\title{
Management and Clinical Utilization of Computed Tomography, Magnetic Resonance Imaging, and Angiography in Hokkaido University Hospital Picture Archiving and Communication System
}

\author{
Kazuo Miyasaka, Satoru Abe, Nobuyuki Fujita, and Kiyoshi Choji
}

\begin{abstract}
We made a preliminary assessment of the Hokkaido University picture archiving and communication system (HU-PACS). Data access time from either imaging machines or data base to workstations was 1.5 minutes, which is great benefit for data communication in routine examinations. Image quality of the work station was estimated in terms of brain computed tomography $(\mathrm{CT})$ and digitized cerebral angiograms. Cerebral infarction was definitely observed on the cathode-ray tube (CRT) monitor of the work station. Although the picture quality of CRT was acceptable, we had to manipulate window level and width for CRT diagnosis of cerebral angiography. Data compression was routinely used without significant degradation of those image quality. Nevertheless, further improvement of maneuverability of the workstation should be considered.
\end{abstract}

Copyright $(1991$ by W.B. Saunders Company

KEY WORDS: computer, picture archiving and communication system.

$\mathbf{M}$ EDICAL IMAGES are occupying increasingly larger space in hospitals, and they are not easily accessible for clinical use and research work. Five to $20 \%$ of them are lost within several years. Since 1982, we have tried to manage medical images as digital data using a digital imaging system, optical disks, local area network (LAN), and computers. Such a digital data management system was recently initiated as a clinical service in our hospital, although it is still experimental and covers only the radiology department and part of the outpatient clinics at present. In our department, nevertheless, interpretation of computed tomography (CT) scans and magnetic resonance imaging (MRI) has been increasingly made on cathode-ray tube (CRT) monitors at

From the Department of Radiology, Hokkaido University School of Medicine and University Hospital, Sapporo, Japan.

Address reprint requests to Kazuo Miyasaka, Department of Radiology, Hokkaido University School of Medicine, N14 W5, Kitaku, Sapporo 060, Japan.

Copyright (C) 1991 by W.B. Saunders Company

0897-1889/91/0404-0108\$03.00/0 workstations instead of from transparent film. We describe the system configuration of the Hokkaido University picture archiving communication system (HU-PACS) and provide a preliminary assessment of the system in the department of radiology, especially relating to $\mathrm{CT}$, digitized angiograms, and data access.

\section{SYSTEM CONFIGURATION \\ Outline of the System}

The system, shown in Fig 1, is based on an optical loop LAN of $100 \mathrm{Mbps}$ with which an Ethernet-type branch LAN of $10 \mathrm{Mbps}$ is connected. Two branch LANs of them are used only for transferring data acquired at each modality, and the other 9 branch LANs are used for data access at workstations; ie, the branch LAN of data input was completely separated from those of data output to relieve traffic during the busy hours of the day.

The image data base (IDP, Fig 1) consists of a processor (MS 4120A), an optical disk library (ODL) containing 48 optical disks as a jukebox, and magnetic disc units (MDU). The memory capacity of data storage system is 96 Gbytes of ODL for long-term archiving of data and 2.4 Gbytes of MDU for quick data access. New image data transferred to the image data processor is divided into image-identifying data (ie, image management data) and the image itself. Subsequently, the former is registered on the image management system processor (ACOS 610MP; IMP, Fig 1) and the latter is stored in the memory of the image data base.

Imaging system inputs include 6 computed radiography (FCR, Fig 1) devices, 3 CT scans, 1 magnetic resonance imaging (MRI) scan, and 1 film digitizer with charge-coupled device (CCD; FIT, in Fig 1). Almost all plain X-ray studies have been digitized by FCR, and others such as contrast examinations will be converted to FCR the next 2 years.

Images are displayed utilizing image workstation (IWS) and image display terminal (IDT). An IWS has two CRT monitors for images with 1,037 


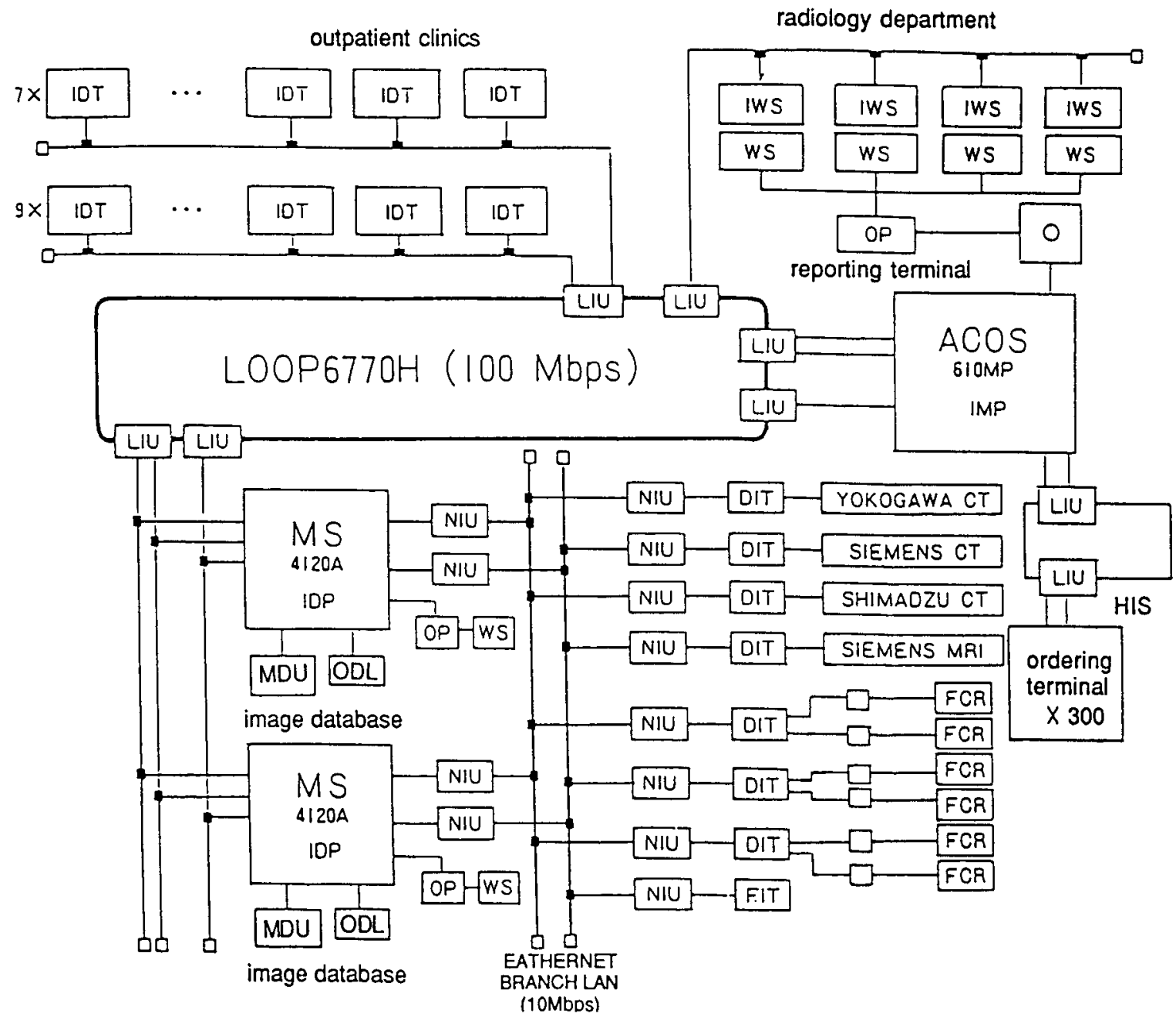

Fig 1. System configuration of HU-PACS. IMP, image management system processor; IDP, image data base processor; IWS, image workstation; IDT, image display terminal; WS, workstation for reporting system; OP, operation processor for reporting system; DIT, digital image input terminal; LIU, loop interface unit; NIU, network interface unit; ODL, optical disk library; MDU, magnetic disk unit; HIS, hospital information system; FIT, film input terminal (film digitizer).

scanning lines and $960 \times 960 \times 9$ display matrices coupled with $32 \mathrm{MB}$ image memory, 1 monitor for image management, and more than 20 functions, including multiimage display of 4 to 36 images. Four IWS are available in the department of radiology for reading images. Sixteen IDT are distributed to the hospital but are limited to the outpatient clinics of orthopedic surgery and general medicine. Laser printers are also available for film output.

The system is linked with hospital information system (HIS) through a HIS host computer (ACOS 610A), in which an extensive relational data base is established, as the ACOS manages patient administrative data, patient laboratory data, and prescriptions. More than 300 HIS ordering terminals are distributed throughout the hospital.

A reporting system has been developed in our department, consisting basically of personal computers, files of image findings, anatomic sites and diagnosis, and a logical diagnostic tree derived as we proceed through the branching frames of the diagnostic menu on the CRT. The advantage of the reporting system is that both the data base of image findings and the diagnostic report to the referring physician are made simultaneously. 

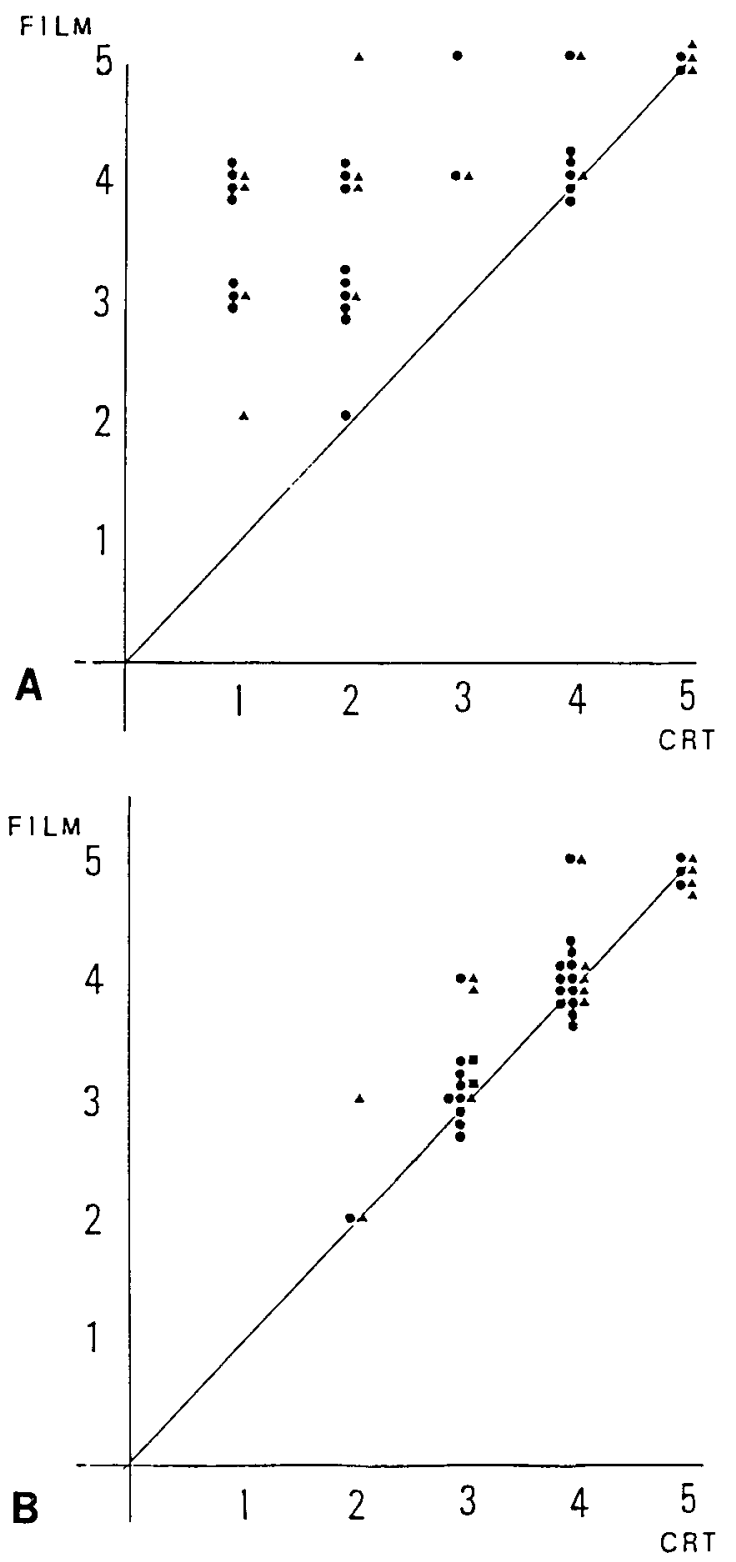

Fig 2. (A and B) Visibility of cerebral perforating arteries on CRT as compared with conventional film (described in text). 1, not visible: 2 , poor; 3 , fair: 4, good; 5 , excellent; $\bullet$, lenticulostriate artery; $\Lambda$, thalamoperiorating artery.

\section{Management and Utilization of HU-PACS}

Data compression by discrete cosine transform is routinely used when data are passed through the digital image input terminal (DIT, Fig 1). CR and digitized film images are transferred at a compression ratio of about 1:10, whereas $\mathrm{CT}$ and MR images are at about 1:3.

Prefetching and downloading of images is performed so that old images for comparison are transferred beforehand from ODL to MDU for expected patients, or comparison images can be called to magnetic disk of the IWS just before the daily conference is held. On the day the patient is examined, image data are automatically transferred to the magnetic disk of the IWS. The reporting system is connected to HIS, and a report can be viewed on the ordering terminal of HIS (currently limited to CT report of brain, head and neck, and spine).

\section{Assessment of the System}

Image quality, data access time, and feasibility of the workstation (IWS) were assessed. Image quality of IWS was evaluated by two experienced neuroradiologists who compared it with conventional transparent film in terms of detectability of cerebral infarction, including small lesion (lacunar infarction) on CT and visibility of cerebral perforating arteries on carotid and vertebral angiograms.

Forty cases of brain CT in which the diagnosis of cerebral infarction had previously been made on transparent film, with the data stored in ODL, were selected for reviewing. The radiology resident selected the cases on the basis of the recorded reports so that the reviewers had no knowledge of the location and extent of the cerebral lesion. Two reviewers observed CT images on CRT independently and described the location and numbers of cerebral lesion (or lesions); they reviewed the transparent films on another day.

For evaluation of cerebral perforating arteries, 39 carotid and vertebral angiograms were selected from our film storage. The original film of each was digitized by a film digitizer at $2000 \times 2000$ $\times 10$ bits. The reviewers noted visibility of the lenticulostriate arteries on anteroposterior (AP) carotid angiograms and of the thalamoperforating arteries on lateral vertebral angiograms. Visibility of the arteries was graded into five steps, depending on the point to which the branch of the arteries could be traced.

Because CT data evaluated on IWS had been compressed at about one third (3:1) of their original size, as had angiograms to about one tenth, the effect of data compression was not excluded in estimating and comparing image quality on CRT and film. Access time of the data was measured because we considered that LAN and CPU would be busy during the day when image data 


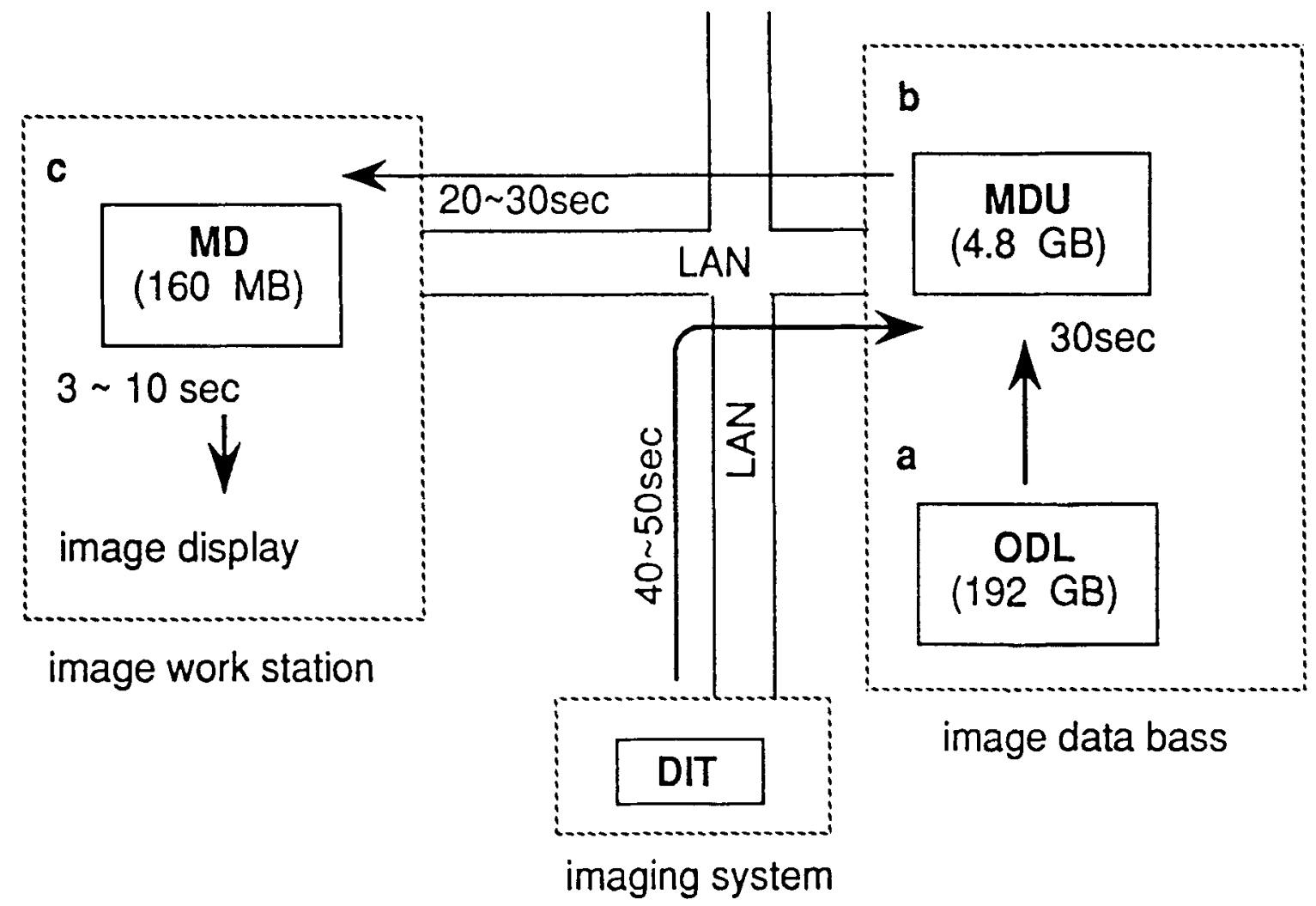

Fig 3. Data flow and average access time. MDU, magnetic disk unit; ODL, optical disk library; DIT, digital image input terminal; LAN, local area network.

were maximally transferred. Function and maneuverability of IWS were also evaluated.

\section{RESULTS AND DISCUSSION}

\section{Image Quality}

Cerebral infarctions in the selected 40 cases were accurately detected on the IWS in all instances. There was no degradation of image quality, even though 3:1 data compression was used.

Visibility of the lenticulostriate and thalamoperforating arteries was much impaired when window level and width were fixed (Fig 2, left). On the other hand, when window level and width were freely changed to optimize the image, visibility of those small arteries was significantly improved, almost to the same degree as that provided by conventional film (Fig 2, right). This result indicates that visibility of the small arteries is affected by the tone and density of an original film before it is digitized but not by the picture quality of IWS itself. Thus, we hope that CR will be adopted for cerebral angiography on CRT diagnosis because $\mathrm{CR}$ offers constant image quality irrespective of variable factors introduced by thickness of objects and exposure dose.

\section{Access Time}

Access time for an old image stored in ODL required only 40 to 60 seconds for display on the IWS even during busy hours of the day. Access time for a new image from image acquisition machines (each modality) required 1.5 minutes through the interface and data base (Fig 3). These access time will be acceptable for clinical utilization of HU-PACS.

The software of the IWS most commonly used were window level and width presetting, multiimage display, magnification, and reference to patient management data. Nevertheless, operation of the IWS is not yet very fast because we routinely use CRT diagnosis in cases that have many images. Furthermore, about 30 seconds was required to display the images of a new patient from the magnetic disk of the IWS after the data were selected by mouse or keyboard. Therefore, further improvement of maneuverability of the IWS should be considered. 